\title{
Multidrug resistant Elizabethkingia meningoseptica bacteremia - Experience from a level 1 trauma centre in India
}

\author{
Aishwarya Govindaswamy ${ }^{1}$, Vijeta Bajpai ${ }^{1}$, Vivek Trikha ${ }^{2}$, Samarth Mittal $^{2}$, \\ Rajesh Malhotra ${ }^{2}$, Purva Mathur,* \\ ${ }^{1}$ Department of Microbiology \& Hospital Infection Control, Jai Prakash Narayan Apex Trauma Centre, All India Institute of \\ Medical Sciences, New Delhi, India; \\ ${ }^{2}$ Department Orthopaedics, Jai Prakash Narayan Apex Trauma Centre, All India Institute of Medical Sciences, New Delhi, India.
}

\begin{abstract}
Summary Elizabethkingia meningoseptica ( $E$. meningoseptica) is a non-fermenting gram negative organism that is commonly detected in the soil and water but is rarely reported to cause human infection. However it is emerging as a nosocomial pathogen in patients admitted in intensive care units (ICUs). Infections caused by this organism have a high mortality rate due to lack of effective therapeutic regimens and its intrinsic resistance to multiple antibiotics. We report our experience in managing Elizabethkingia meningoseptica (E. meningoseptica) septicemia in our ICU patients with septic shock during prolonged intensive care management. Over a two year period four cases were admitted into the polytrauma ICU developed sepsis due to $E$. meningoseptica. All these patients were on mechanical ventilation, had central venous catheter (CVC) and were exposed to various broad spectrum antibiotics. Of the four patients, three died and one recovered. $E$. meningoseptica infection should be considered as a possible etiological agent of sepsis in patients who do not respond to empirical therapy, as this results in an inappropriate choice of antimicrobial therapy, leading to increased morbidity and mortality of patients. Its unusual resistance pattern along with inherent resistance to colistin makes this organism difficult to treat unless susceptibility patterns are available.
\end{abstract}

Keywords: Elizabethkingia meningoseptica (E. meningoseptica), septicaemia, Intensive Care Unit (ICU), multi-drug resistance

\section{Introduction}

Elizabethkingia species are gram negative bacilli which are aerobic, oxidase positive, indole positive, nonmotile bacilli and does not ferment glucose. They can be found commonly in freshwater, saltwater, soil and in hospital environments (1). They do not normally exists in the human body, but have been reported to cause various invasive infections like meningitis, pneumonia, endocarditis, and bacteremia in adults and neonates in association with severe underlying illness (2). The risk factors associated with acquisition of

Released online in J-STAGE as advance publication August 28, 2018.

*Address correspondence to:

Dr. Purva Mathur, Department of Microbiology \& Hospital Infection Control, Jai Prakash Narayan Apex Trauma Centre, All India Institute of Medical Sciences, New Delhi 110029, India.

E-mail: purvamathur@yahoo.co.in this infection include immunosuppression, underlying medical diseases, prolonged hospital stay, prior use of higher antibiotics, indwelling central venous catheter and other invasive devices (3). This organism is resistant to many antibiotics like beta-lactam antibiotics, aminoglycosides, tetracyclines, and chloramphenicol, However, it is susceptible to some agents used to treat Gram-positive bacteria like erythromycin, clindamycin, rifampicin, trimethoprim-sulfamethoxazole, quinolones and vancomycin (4). Selecting appropriate antimicrobial agents for patients infected with E. meningoseptica is difficult due to lack of data on clinical response to different treatments and also due to multiple drug resistance. In this report, we describe a series of four cases with E. meningoseptica bacteraemia and septic shock admitted to the ICUs of our level 1 Trauma Centre.

\section{Patients and Methods}

This is a retrospective study conducted over a two 
year period from June 2016 - May 2018 at our level 1 Trauma Centre. During the above time frame, four cases admitted in the polytrauma ICU developed sepsis due to Elizabethkingia meningoseptica. Blood cultures of these patients grew E. meningoseptica which were identified up to the species level by VITEK 2 GN card (version 7.02, BioMérieux, Inc. Durham, USA). Antimicrobial susceptibility testing was performed by Kirby-Bauer disc diffusion method on Muller Hinton agar and by Vitek 2 (BioMe'rieux) system. The MICs were interpreted based on the Clinical and Laboratory Standard Institute (CLSI) criteria for other nonEnterobacteriaceae. Patient's details and clinical data included sex, age, diagnosis at admission, presence of central venous catheter, dates and dosages of antibiotic drug administration, dates of catheter removal, and outcome of bacteraemia were recorded. The reports of culture of other samples were also recorded.

\subsection{Case 1}

A 54 year old male patient admitted with history of Road Traffic Accident (RTA), presented with loss of consciousness during admission. On examination, the patient was intubated from an outside hospital, stable haemodynamically, the Glasgow Coma Scale (GCS) being E4VTM5. The case was suspected to have head injury with chest trauma, abdominal injury and pelvic fracture along with fracture of long bones (Polytrauma). On local examination of the right leg, the patient had swelling, tenderness and deformity. $\mathrm{X}$-ray finding revealed intertrochantric fracture of femur following which the patient was started on intravenous (IV) augmentin 1.2 gram and injection metrogyl 750mg IV stat and operated on an emergency basis and was shifted to polytrauma ICU. On $3^{\text {rd }}$ postoperative day in the ICU, the patient developed high grade fever along with pus from the operated site. The pus culture grew Acinetobacter baumannii sensitive to colistin and tigecycline. Based on the culture reports, the patient was started on colistin 4.5 million IU IV BD and meropenem 1 gram IV TDS for 14 days. Despite two weeks of antibiotic treatment, the patient still had persistent fever. The antibiotic was changed to IV tigecycline $50 \mathrm{mg}$ BD. Total leucocyte count (TLC) was 13,350 cells $/ \mathrm{mm}^{3}(4,500$ - 11,000), hemoglobin $9.5 \mathrm{~g} /$ dL (13 - 15), sodium 136 meq/L (145 - 150), potassium $4.8 \mathrm{meq} / \mathrm{L}(3.5-5.5)$ and chlorides were $105 \mathrm{meq} /$ L (96 - 106). The symptoms persisted with increasing TLC counts, thrombocytopenia and raised procalcitonin levels $2.53 \mathrm{ng} / \mathrm{mL}$ (normal < 0.15 ) suggestive of sepsis. Two sets of blood cultures were sent, one from central line and one from peripheral line. Both the cultures grew E. meningoseptica which was resistant to most of the antibiotics. The MICs of various antibiotics are shown in Table 1. The patient was started on IV levofloxacin and inotropic support. Despite all possible resuscitative measures the patient had a fatal outcome on the $42^{\text {nd }}$ day of hospitalization.

\subsection{Case 2}

A 30 year old female was admitted in our emergency department following history of fall due to which she had sustained injury on the right side of the hip. On local examination there was tenderness, swelling, painful and restricted movements. X-ray revealed intertrochanteric fracture with proximal femoral nail (PFNA) in situ. The patient was started on injection cefoperazone/sulbactum $2 \mathrm{gm}$ IV BD, injection levofloxacin $750 \mathrm{gm}$ IV OD. She was operated on an elective basis and shifted to the orthopaedic ward. During the $14^{\text {th }}$ postoperative day, the patient developed fever, hypotension, and generalised edema with tachycardia and was shifted to the ICU. In the ICU, the antibiotics were escalated to injection colistin 1 million units IV TDS and injection meropenem 1 gm IV TDS. Multiple blood cultures were sent. The blood culture sent on the $13^{\text {th }}$ day of ICU stay grew E. meningoseptica sensitive to cefepime-tazobactum and piperacillin-

Table 1. Antimicrobial susceptibility profile of $E$. meningoseptica isolated from four cases

\begin{tabular}{|c|c|c|c|c|}
\hline \multirow{2}{*}{ Antibiotics } & \multicolumn{4}{|c|}{ Minimum Inhibitory concentration (MIC) } \\
\hline & Isolate 1 & Isolate 2 & Isolate 3 & Isolate 4 \\
\hline Amikacin & $\geq 64(\mathrm{R})$ & $\geq 64(\mathrm{R})$ & $\geq 64(\mathrm{R})$ & $\geq 64(\mathrm{R})$ \\
\hline Gentamicin & $\geq 16(\mathrm{R})$ & $\geq 16(\mathrm{R})$ & $\geq 16(\mathrm{R})$ & $\geq 32(\mathrm{R})$ \\
\hline Cefepime & $\geq 64(\mathrm{R})$ & $\geq 32(\mathrm{R})$ & $\geq 64(\mathrm{R})$ & $\geq 64(\mathrm{R})$ \\
\hline Cefoperazone & $\geq 64(\mathrm{R})$ & $\geq 64(\mathrm{R})$ & $\geq 32(\mathrm{I})$ & $\geq 64(\mathrm{R})$ \\
\hline Ceftazidime & $\geq 32(\mathrm{R})$ & $\geq 64(\mathrm{R})$ & $\geq 64(\mathrm{R})$ & $\geq 64(\mathrm{R})$ \\
\hline Ciprofloxacin & $\geq 4(\mathrm{R})$ & $\geq 4(\mathrm{R})$ & $\geq 4(\mathrm{R})$ & $0.5(\mathrm{~S})$ \\
\hline Levofloxacin & $\geq 4(\mathrm{I})$ & $\geq 8(\mathrm{R})$ & $\geq 8(\mathrm{R})$ & $0.5(\mathrm{~S})$ \\
\hline Imipenem & $\geq 16(\mathrm{R})$ & $\geq 16(\mathrm{R})$ & $\geq 16(\mathrm{R})$ & $\geq 16(\mathrm{R})$ \\
\hline Meropenam & $\geq 16(\mathrm{R})$ & $\geq 16(\mathrm{R})$ & $\geq 16(\mathrm{R})$ & $\geq 16(\mathrm{R})$ \\
\hline Piperacillin & $\geq 128(\mathrm{R})$ & $\geq 64(\mathrm{I})$ & $\geq 128(\mathrm{R})$ & $\geq 128(\mathrm{R})$ \\
\hline Piperacillin/Tazobactam & $\geq 128(\mathrm{R})$ & $\leq 16(\mathrm{~S})$ & $\geq 128(\mathrm{R})$ & $\geq 128(\mathrm{R})$ \\
\hline Ticarcillin/Clavulanic acid & $\geq 128(\mathrm{R})$ & $\geq 128(\mathrm{R})$ & $\leq 16(\mathrm{~S})$ & $\geq 128(\mathrm{R})$ \\
\hline Trimethoprim/Sulphamethoxazole & $\geq 320(\mathrm{R})$ & $\geq 160(\mathrm{R})$ & $\geq 160(\mathrm{R})$ & $\leq 2(\mathrm{~S})$ \\
\hline
\end{tabular}


tazobactum. The antibiotic was changed to piperacillintazobactum based on the susceptibility report. On the $19^{\text {th }}$ day of ICU stay, the patient again had an episode of fever, hypotension and also sustained a cardiac arrest. The patient was started on multiple inotropes, but could not be revived.

\subsection{Case 3}

A 76 year old female had a history of fall following which she sustained injury to the left hip. She was admitted to our emergency department. Local examination revealed pain, swelling and deformity of the left thigh. X-ray revealed sub trochanteric fracture of left femur. The patient was a known case of hypertension and Type 2 diabetes mellitus and had deranged kidney and cardiac parameters and was investigated for surgical clearance. Following surgical clearance the patient was operated on and owing to high risk and underlying illness, she was shifted to the ICU. On the $5^{\text {th }}$ postoperative day, she developed high grade fever and the blood cultures grew Escherichia coli sensitive to tigecycline and colistin. The patient was started on the above antibiotics as per the culture report. Despite these antibiotics the patient continued to have fever and the blood cultures sent on the $15^{\text {th }}$ day of ICU stay grew Enterococcus faecium sensitive to ampicillinsulbactum, vancomycin, teicoplanin and linezolid. The patient was started on linezolid $600 \mathrm{mg}$ IV BD. The patient still persisted to have fever on and off. The blood cultures sent on the $31^{\text {st }}$ day of hospitalisation grew E. meningoseptica sensitive to cefepimetazobactum, cefoperazone-sulbactum, piperacillintazobactum and ticarcillin-clavulanate. The patient was started on cefepime-tazobactum. Despite all the efforts the patient succumbed to death on the $47^{\text {th }}$ day of ICU stay.

\subsection{Case 4}

A 16 year old male was admitted with a history of RTA following which he presented with loss of consciousness and vomiting. He was suspected to have head injury. The CT scan revealed left temporal and occipital contusion with mass effect and midline shift. The patient was started on augmentin $1.2 \mathrm{mg}$ IV stat. An emergency decompressive craniectomy was done and he was shifted to the ICU. Post operatively, the patient was started on injection cefoperazone-sulbactum $1 \mathrm{gm} \mathrm{IV} \mathrm{BD} \mathrm{and} \mathrm{injection} \mathrm{netilmycin} 200 \mathrm{mg}$ IV OD and was kept under observation in the ICU. On the $5^{\text {th }}$ postoperative day the patient developed fever along with chest infiltrates, following which endotracheal aspirate (ETA) sample was sent to the lab. The ETA grew Pseudomonas aeruginosa which was sensitive only to colistin. The patient was then started on IV colistin. The patient still continued to have fever with increasing TLC counts. Multiple blood culture samples were sent. The blood sample sent on the $9^{\text {th }}$ day of ICU stay grew Elizabethkingia meningosepticum sensitive to ciprofloxacin, levofloxacin and trimethoprim/ sulphamethoxazole. The MIC values of the antibiotics are shown in Table 1. Prompt treatment with ciprofloxacin led to improvement of the patient.

\section{Results and Discussion}

Four cases of E. meningoseptica infections were reported in our ICU from June 2016 - May 2018. All four patients blood samples grew E. meningoseptica. The colony morphology of E.meningosepticum was smooth circular 1-2 mm colonies with entire edges and regular margins with a slight yellow pigmentation on nutrient agar after 24 hours incubation. The growth on blood agar showed 1-2 mm smooth, circular, greyish-white non-hemolytic colonies. There was no growth on MacConkey agar. All isolates grew at a temperature of $37^{\circ} \mathrm{C}$.

Among the four patients, three patients were immunocompetent, one was immunocompromised with underlying illness. The mean age of the patients was 43.7 years. Two were males and two were females. The median time from admission to isolation of $E$. meningoseptica was 12 days range (10-25 days). Two of them had long bone fracture, one had multiple rib

Tables 2. Summary of patients with Elizabethkingia meningoseptica infections

\begin{tabular}{|c|c|c|c|c|c|c|c|c|}
\hline Case & Age & Sex & Diagnosis & $\begin{array}{l}\text { Underlying } \\
\text { illness }\end{array}$ & $\begin{array}{l}\text { Length } \\
\text { of stay }\end{array}$ & $\begin{array}{l}\text { Previous antibiotic } \\
\text { exposure }\end{array}$ & $\begin{array}{l}\text { Antibiotic } \\
\text { treatment }\end{array}$ & Outcome \\
\hline 1 & 53 & $\mathrm{M}$ & $\begin{array}{l}\text { Hemopneumothorax } \\
\text { with septic shock }\end{array}$ & Nil & 42 & $\begin{array}{l}\text { Meropenam, } \\
\text { Tigecycline Colistin, }\end{array}$ & Levofloxacin & Death \\
\hline 2 & 30 & $\mathrm{~F}$ & $\begin{array}{l}\text { Intertrochanteric fracture } \\
\text { femur with septic shock }\end{array}$ & Nil & 55 & $\begin{array}{l}\text { Cefeperazone sulbactum, } \\
\text { Levofloxacin, } \\
\text { Meropenam Colistin, }\end{array}$ & $\begin{array}{l}\text { Piperazillin/ } \\
\text { Tazobactum }\end{array}$ & Death \\
\hline 3 & 76 & $\mathrm{~F}$ & $\begin{array}{l}\text { Subtrochanteric fracture } \\
\text { femur with sepsis }\end{array}$ & $\begin{array}{c}\text { HT, } \\
\text { Type } 2 \text { DM }\end{array}$ & 63 & Colistin & $\begin{array}{l}\text { Cefepime- } \\
\text { tazobactum }\end{array}$ & Death \\
\hline 4 & 16 & M & Head Injury with sepsis & Nil & 15 & $\begin{array}{l}\text { Cefeperazone-sulbactum, } \\
\text { Netilmicin }\end{array}$ & Ciprofloxacin & Survived \\
\hline
\end{tabular}


fractures with hemopneumothorax, and one had head injury with acute Extra Dural Haematoma (EDH). Among the four, two of them underwent surgical procedures for long bone fracture and one underwent surgical evacuation of EDH. All four patients had history of a recent hospitalization. At the time of bacteremia, all four patients were in the ICU and required mechanical ventilation. All four patients had a central line at the time of bacteremia. All the patients received combination therapy with two or more antibiotics, which included cefeperazonesulbactum, meropenem, tigecycline and colistin. Of the four patients, three died and one recovered. The clinical features and risk factors of all the patients are summarized in Table 2. Antimicrobial susceptibility of all the isolates was performed and the minimum inhibitory concentration (MICs) of the isolates are shown in Table 1. All four isolates were resistant to most of the antibiotics commonly use in the ICUs. Among the four only one isolate tested was susceptible to trimethoprim-sulfamethoxozole, ciprofloxacin, and levofloxacin.

E. meningoseptica is capable of survival in hospital environments. The source of the organism is most likely from contaminated water supply (since it survives chlorine treatment) or from hospital equipment and other sources such as saline solutions used for flushing procedures, disinfectants, hands of hospital staffs, infant formulas etc. (5). The risk factors for infection by this organism includes diabetes mellitus, steroid use, malignancies, organ transplantation, neutropenia, prolonged hospitalisation, prior exposure to multiple antibiotics, immunocompromised host and chronic haemodialysis (6). In our study, we observed that prolonged hospital stay especially in the ICUs, presence of central venous catheter (CVC), exposure to various broad spectrum antibiotics, presence of shock and recent surgery were the major risk factors. A similar study conducted in Taiwan showed that $60 \%$ of the infection were acquired in the ICUs (7). In our study too, all four patients were in the ICUs with CVC in place at the time of development of bacteraemia. One of the patients had diabetes mellitus as an underlying risk factor.

Elizabethkingia spp are known to be intrinsically resistant to tigecycline and polymyxins due to the production of both class A extended spectrum beta lactamases (ESBL) and class B metallo- $\beta$-lactamases (MBLs). The MBLs confer resistance to aztreonam and carbapenems, the latter being the mainstay drugs for the treatment of multidrug-resistant Gram negative bacteria (8). A study conducted by Khan et al. has shown that previous exposure to Gram negative antibiotic cover is known to predispose to hospital infections caused by Elizabethkingia spp. (9). Our study also showed similar findings where all four patients had received prolonged gram negative antimicrobial cover in the ICUs.
Infections caused by Elizabethkingia spp. are very difficult to treat and have a high mortality rate especially in immunocompromised patients. A study conducted by Boroda $\mathrm{K}$ et al. reported a 28 day mortality rate of $41 \%$ for nosocomial infections and $9.1 \%$ for community acquired infections (10).

We also observed a high mortality, where three out of four cases had a fatal outcome. Presence of shock and use of inappropriate antibiotics acted as independent risk factors for mortality as seen in the other studies (11). Delay in the identification of the organism may lead to treatment failure. Different AST methods gives varied susceptibility results, further complicating its management. The disc diffusion methods are considered to be unreliable and broth micro dilution is the preferred method. There are no standard guidelines for empiric treatment of E. meningoseptica infection and most authors suggest using antimicrobial agents based on the minimal inhibitory concentration results from properly performed susceptibility tests (12).

To conclude, Elizabethkingia meningoseptica is an emerging pathogen in trauma victims who undergo various surgical procedures. It is essential to keep a high index of suspicion for this infection since failure to start the appropriate antibiotic therapy may lead to a fatal outcome. In the hospital setting, strict contact isolation should be implemented to prevent colonisation of individuals and outbreak.

\section{Acknowledgements}

I wish to acknowledge my laboratory staff and technicians for helping in laboratory testing and identification of isolates.

\section{References}

1. Shinha T, Ahuja R. Bacteremia due to Elizabethkingia meningoseptica. IDCases. 2015; 2:13-15.

2. Han MS, Kim H, Lee Y, Kim M, Ku NS, Choi JY, Yong D, Jeong SH, Lee K, Chong Y. Relative prevalence and antimicrobial susceptibility of clinical isolates of Elizabethkingia species based on 16S rRNA gene sequencing. J Clin Microbiol. 2016; 55:274-280.

3. Tak V, Mathur P, Varghese P, Misra MC. Elizabethkingia meningoseptica: An emerging pathogen causing meningitis in a hospitalized adult trauma patient. Indian J Med Microbiol. 2013; 31:293-295.

4. Jung SH, Lee B, Mirrakhimov AE, Hussain N. Septic shock caused by Elizabethkingia meningoseptica: A case report and review of literature. BMJ Case Rep. 2013; 2013. pii:bcr2013009066.

5. Rastogi N, Mathur P, Bindra A, Goyal K, Sokhal N, Kumar S, Sagar S, Aggarwal R, Soni KD, Tandon V. Infections due to Elizabethkingia meningoseptica in critically injured trauma patients: A seven-year study. J Hosp Infect. 2016; 92:30-32.

6. Ceyhan M, Yildirim I, Tekeli A, Yurdakok M, Us E, Altun B, Kutluk T, Cengiz AB, Gurbuz V, Barin C, Bagdat A, Cetinkaya D, Gur D, Tuncel O. A 
Chryseobacterium meningosepticum outbreak observed in 3 clusters involving both neonatal and non-neonatal pediatric patients. Am J Infect Control. 2008; 36:453457.

7. Lin YT, Chiu CH, Chan YJ, Lin ML, Yu KW, Wang FD, Liu CY. Clinical and microbiological analysis of Elizabethkingia meningoseptica bacteremia in adult patients in Taiwan. Scand J Infect Dis. 2009; 41:628634.

8. Ceyhan M, Celik M. Elizabethkingia meningosepticum (Chryseobacterium meningosepticum) infections in Children. Int J Pediatr. 2011; 215237.

9. Khan ID, Lall M, Sen S, Ninawe SM, Chandola P. Multiresistant Elizabethkingia meningoseptica infections in tertiary care. Med J Armed Forces India. 2015; 71:282-286.

10. Boroda K, Li L. Elizabethkingia meningosepticum in a patient with six-year bilateral perma-catheters. Case Rep Infect Dis. 2014; 985306.

11. Swain B, Rout S, Otta S, Rakshit A. Elizabethkingia meningoseptica: An unusual cause for septicaemia. JMM Case Rep. 2015; doi:10.1099/jmmcr.0.000005

12. Bennett JE, Dolin R, Blaser MJ. Mandell, Douglas, and Bennett's Principles and Practice of Infectious Diseases 8th Edition. Elsevier Health Sciences; 2014; pp 293.

(Received July 27, 2018; Revised August 16, 2018; Accepted August 19, 2018) 